\title{
Dietary Strategies to Optimize Wound Healing after Periodontal and Den- tal Implant Surgery: An Evidence-Based Review
}

\author{
Beatrice Y. Lau ${ }^{1}$, Bryan D. Johnston ${ }^{1}$, Peter C. Fritz ${ }^{2}$ and Wendy E. Ward ${ }^{1, *}$ \\ ${ }^{1}$ Center for Bone and Muscle Health, Faculty of Applied Health Sciences, Brock University, St. Catharines, Ontario, \\ Canada \\ ${ }^{2}$ Reconstructive Periodontics and Implant Surgery Clinic, Fonthill, Ontario, Canada; Staff Periodontist, Niagara Health \\ System; and Center for Bone and Muscle Health, Faculty of Applied Health Sciences, Brock University, St. Catharines, \\ Ontario, Canada
}

\begin{abstract}
Methods to optimize healing through dietary strategies present an attractive option for patients, such that healing from delicate oral surgeries occurs as optimally as possible with minimal patient-meditated complications through improper food choices. This review discusses findings from studies that have investigated the role of diet, either whole foods or individual dietary components, on periodontal health and their potential role in wound healing after periodontal surgery. To date, research in this area has largely focused on foods or individual dietary components that may attenuate inflammation or oxidant stress, or foster de novo bone formation. These studies suggest that a wide variety of dietary components, including macronutrients and micronutrients, are integral for optimal periodontal health and have the potential to accelerate oral wound healing after periodontal procedures. Moreover, this review provides guidance regarding dietary considerations that may help a patient achieve the best possible outcome after a periodontal procedure.
\end{abstract}

Keywords: Anti-inflammation, anti-oxidants, dietary strategies, implant surgery, minerals, oral wound healing, periodontal surgery, vitamins.

\section{INTRODUCTION}

Periodontal and dental implant surgeries typically involve invasive techniques with possible post-operative complications that may delay healing times and subsequently negatively affect a successful clinical outcome. The duration of the surgical procedure, the patient's physical anatomy, health condition, and smoking status, as well as the clinician's skill, will in turn affect the rates of healing of the surrounding periodontal and bone tissues. However, often overlooked is the patient's role in healing during the postoperative period, as mastication carries inherent risks not typical for surgeries in other parts of the body and patients often adopt a restricted soft diet with limited nutrition.

Methods to optimize healing through dietary strategies present an attractive option for patients, such that healing from delicate oral surgeries occurs as optimally as possible with minimal patient-meditated complications through improper food choices. Although the application of growth factor-containing agents in osseous grafts and collagen gels is a common method for periodontal wound healing [1], specialized dietary strategies pre- and post-surgery may provide an ideal approach to optimizing healing after dental implant and periodontal surgery, particularly since such a healthful

*Address correspondence to this author at the Center for Bone and Muscle Health, Faculty of Applied Health Sciences, Brock University, 500 Glenridge Ave. St. Catharines, Ontario, L2S 3A1, Canada;

Tel: 9056885550 X3024; Fax: 905688 6840; E-mail: wward@brocku.ca diet would benefit overall health as well as oral health. The dietary strategy must also take into account the swelling of the tissues post-surgery as well as extreme temperatures, which may aggravate hypersensitive teeth or promote edema and hemorrhage. The consistency of the diet must also restrict trauma to the incisions, and in some cases account for an edentulous situation as with overdenture implant surgery. In order to develop such strategies, it is imperative to review studies to date that have investigated the relationship between dietary components and oral wound healing. Moreover, because of the paucity of data in this area, a review of the evidence regarding dietary components and periodontal health is useful to more fully understand how nutrients either in foods or as dietary supplements - contribute to overall periodontal health. This review provides an up to date synopsis of these studies and discussion of dietary considerations that may help a patient achieve the best possible outcome after a periodontal procedure.

\section{THE ROLE OF VITAMIN SUPPLEMENTS IN ORAL WOUND HEALING}

Few intervention studies have investigated if and how specific dietary components modulate oral wound healing [2-5] (Table 1). One study showed that vitamin B12 supplementation resulted in lower pain scores at 6 and 120 hours postextraction with no effect on facial swelling [2]. Among patients undergoing surgical removal of an oral tumor, a selenium supplement was associated with a reduction in associated-lymphedema [3]. As expected with lymphedema, there 
Table 1. Studies Investigating the Effects of Dietary Components on Post-Operative Outcomes in Adults

\begin{tabular}{|c|c|c|c|c|c|c|c|}
\hline \multirow[t]{2}{*}{$\begin{array}{c}\text { Dietary } \\
\text { Component } \\
\text { [Reference] }\end{array}$} & \multirow[t]{2}{*}{$\begin{array}{l}\text { Study } \\
\text { Type }\end{array}$} & \multicolumn{2}{|c|}{$\begin{array}{c}\text { Characteristics of } \\
\text { Participants }\end{array}$} & \multirow[t]{2}{*}{$\begin{array}{c}\text { Intervention } \\
\text { Duration }\end{array}$} & \multicolumn{2}{|c|}{ Intervention Groups } & \multirow[t]{2}{*}{ Post-Operative Outcomes } \\
\hline & & $\mathbf{n}$ & $\begin{array}{c}\text { Surgical } \\
\text { Procedure/ } \\
\text { Clinical Condition }\end{array}$ & & Control & Treatment & \\
\hline $\begin{array}{l}\text { Vitamin B12 } \\
{[2]}\end{array}$ & $\begin{array}{l}\text { Double- } \\
\text { blind, } \\
\text { random- } \\
\text { ized } \\
\text { study }\end{array}$ & 80 & $\begin{array}{l}\text { Mandibular third } \\
\text { molar extraction in } \\
\text { healthy patients, no } \\
\text { medication in previ- } \\
\text { ous } 3 \text { months that } \\
\text { might affect inflam- } \\
\text { matory responses, no } \\
\text { systemic diseases, } \\
\text { no alcohol, non- } \\
\text { smokers }\end{array}$ & $\begin{array}{l}\text { One tablet } 30 \\
\text { min post- } \\
\text { operation and } \\
\text { for } 4 \mathrm{~d} \text {, once } \\
\text { daily }\end{array}$ & $\begin{array}{c}20 \mathrm{mg} \\
\text { piroxicam }\end{array}$ & $\begin{array}{l}2.5 \mathrm{mg} \text { cyanocobala- } \\
\text { min with } \\
10 \mathrm{mg} \text { piroxicam, } \\
1 \mathrm{mg} \text { dexamethasone, } \\
35 \mathrm{mg} \text { orphenadrine } \\
\text { citrate }\end{array}$ & $\begin{array}{l}\text { Lower pain scores at } 6 \mathrm{~h} \text { and } 120 \mathrm{~h} \\
\text { post-extraction in treatment group. } \\
\text { No effect on facial swelling. }\end{array}$ \\
\hline $\begin{array}{l}\text { Selenium } \\
\text { [3] }\end{array}$ & $\begin{array}{l}\text { Double- } \\
\text { blind, } \\
\text { random- } \\
\text { ized } \\
\text { study }\end{array}$ & 20 & Oral tumour surgery & $\begin{array}{l}3 \text { wk, once } \\
\text { daily }\end{array}$ & Placebo & $\begin{array}{l}1000 \mu \mathrm{g} \text { sodium } \\
\text { selenite through IV or } \\
\text { oral route }\end{array}$ & $\begin{array}{l}\text { Inverse correlation between lym- } \\
\text { phedema severity and whole } \\
\text { blood/plasma selenium concentra- } \\
\text { tion and glutathione peroxidase } \\
\text { activity. Positive correlation be- } \\
\text { tween ROS concentration and ex- } \\
\text { tent of lymphedema. Significant } \\
\text { reduction of lymphedema in treated } \\
\text { group. }\end{array}$ \\
\hline $\begin{array}{l}\text { Vitamin B } \\
\text { complex } \\
{[4]}\end{array}$ & $\begin{array}{l}\text { Double- } \\
\text { blind, } \\
\text { random- } \\
\text { ized } \\
\text { study }\end{array}$ & 30 & $\begin{array}{l}\text { Access flap surgery } \\
\text { in patients with } \\
\text { generalized moder- } \\
\text { ate to severe chronic } \\
\text { periodontitis, } \geq 2 \\
\text { teeth in same sextant } \\
\text { with probing depth } \geq \\
5 \text { mm and bleeding } \\
\text { upon probing }\end{array}$ & $\begin{array}{l}30 \text { d post- } \\
\text { operation, } \\
\text { once daily }\end{array}$ & Placebo & $\begin{array}{c}50 \mathrm{mg} \text { each of } \\
\text { thiamine, riboflavin, } \\
\text { niacinaide, } \\
\text { pantothenate and } \\
\text { pyridoxine; } \\
50 \mu \mathrm{g} \text { each of biotin } \\
\text { and cyanocobalamin; } \\
400 \mu \mathrm{g} \text { of folate }\end{array}$ & $\begin{array}{l}\text { Better clinical attachment level in } \\
\text { vitamin B complex supplemented } \\
\text { group. No difference in gingival } \\
\text { index, plaque index or bleeding } \\
\text { upon probing between groups. }\end{array}$ \\
\hline $\begin{array}{l}\text { Vitamin D } \\
\text { [5] }\end{array}$ & $\begin{array}{l}\text { Double- } \\
\text { blind, } \\
\text { random- } \\
\text { ized } \\
\text { study }\end{array}$ & 40 & $\begin{array}{l}\text { Open flap debride- } \\
\text { ment surgery in } \\
\text { patients with severe } \\
\text { periodontal disease }\end{array}$ & $\begin{array}{l}3 \text { days pre- } \\
\text { surgery, } \\
\text { continued } \\
\text { daily for } 6 \\
\text { wks }\end{array}$ & $\begin{array}{l}\text { Placebo, } \\
1000 \mathrm{mg} \\
\text { calcium, } \\
800 \mathrm{IU} \\
\text { vitamin D } \\
\text { daily }\end{array}$ & $\begin{array}{l}20 \mu \mathrm{g} \text { teriparatide, } \\
1000 \mathrm{mg} \mathrm{Ca}, 800 \mathrm{IU} \\
\text { vitamin D daily }\end{array}$ & $\begin{array}{l}\text { CAL and PDR in vitamin D suffi- } \\
\text { cient }(>20 \mathrm{ng} / \mathrm{mL} \text { serum } 25(\mathrm{OH}) \mathrm{D}) \\
\text { patients. Vitamin D sufficient pa- } \\
\text { tients receiving teriparatide experi- } \\
\text { enced better: CAL at } 6 \text { mo, PDR at } \\
3,6 \text {, and } 9 \text { mo, and RLBG at } 6,9 \text {, } \\
\text { and } 12 \text { mo compared to vitamin D } \\
\text { insufficient patients also receiving } \\
\text { teriparatide. }\end{array}$ \\
\hline
\end{tabular}

$\mathrm{Ca}$, calcium; CAL, clinical attachment level; PDR, probing depth reduction; RLBG, radiographic linear bone gain; ROS, reactive oxygen species

was a positive correlation between extent of lymphedema and reactive oxygen species (ROS) concentration. Moreover, there was an inverse correlation between lymphedema severity and circulating selenium concentration and glutathione peroxidase activity. This suggests that selenium may have attenuated lymphedema via an antioxidant mechanism such as elevated glutathione peroxidase activity. In patients with generalized moderate to severe chronic periodontitis who had undergone access flap surgery, a vitamin B complex given daily for 30 days post-operation led to greater clinical attachment levels than a placebo, although there was no effect on gingival index, plaque index and bleeding on probing [4]. Another study showed that vitamin $\mathrm{D}$ status - determined by measurement of serum $25(\mathrm{OH}) \mathrm{D}$ - was associated with better post-operative surgical outcome. Among patients with severe chronic periodontitis, vitamin D sufficiency (serum 25(OH)D of $>20$ $\mathrm{ng} / \mathrm{mL}$ ) before periodontal surgery was associated with improved clinical attachment levels and reduced probing depth for up to 12 months after surgery [5]. In contrast, patients who were vitamin D deficient (serum 25(OH)D of $<20 \mathrm{ng} / \mathrm{mL}$ ), prior to periodontal surgery did not experience clinical benefits with the 6 week supplementation of vitamin D (800 IU) and calcium $(1000 \mathrm{mg})$. However, as noted by the authors, a longer supplementation period, i.e. 3 months or more, is required to observe elevations in serum 25(OH)D [6]. A subset of patients who were vitamin D sufficient and taking teriparatide (parathyroid hormone), experienced more bone gain from 6 to 12 months after surgery. This would be expected given the anabolic nature of teriparatide. While mechanisms were not identified, vitamin D is known to have immunomodulatory functions in addition to its well-established role in regulation of calcium metabolism. These studies provide some evidence to suggest that certain minerals and vitamins improve post-surgical outcomes. Exact mechanisms are not known, but it can be speculated based on potential antioxidant properties of selenium while the B vitamins are integral to 
Table 2. Studies Relating Dietary Components and Periodontal Health in Adults

\begin{tabular}{|c|c|c|c|c|c|c|c|c|}
\hline \multirow[t]{2}{*}{$\begin{array}{c}\text { Dietary } \\
\text { Component } \\
\text { [Reference] }\end{array}$} & \multirow[t]{2}{*}{$\begin{array}{l}\text { Study } \\
\text { Type }\end{array}$} & \multicolumn{3}{|c|}{ Characteristics of Participants } & \multirow[t]{2}{*}{$\begin{array}{l}\text { Intervention } \\
\text { Duration }\end{array}$} & \multicolumn{2}{|c|}{$\begin{array}{c}\text { Intervention/Comparison } \\
\text { Groups }\end{array}$} & \multirow[t]{2}{*}{ Outcomes } \\
\hline & & Number & Age & $\begin{array}{l}\text { Clinical } \\
\text { Condition }\end{array}$ & & Control & Treatment & \\
\hline \multicolumn{9}{|c|}{ Macronutrients with Potential Anti-Inflammatory Activity } \\
\hline $\begin{array}{l}\text { Saturated fat, } \\
\text { PUFA, choles- } \\
\text { terol } \\
{[10]}\end{array}$ & $\begin{array}{l}\text { Prospec- } \\
\text { tive, ran- } \\
\text { domized } \\
\text { study }\end{array}$ & $\begin{array}{l}250 \text { par- } \\
\text { ents from } \\
148 \text { fami- } \\
\quad \text { lies }\end{array}$ & $\begin{array}{l}\text { Mean } \\
\text { age: } \\
34.2 \text { y }\end{array}$ & & $29 \mathrm{mo}$ & $\begin{array}{l}\text { "Normal } \\
\text { diet" }\end{array}$ & $\begin{array}{c}\text { Anti- } \\
\text { atherosclerotic } \\
\text { diet (high } \\
\text { PUFA/SFA, low } \\
\text { SFA, low serum } \\
\text { cholesterol, high } \\
\text { HDL) }\end{array}$ & $\begin{array}{l}\text { NS differences in periodontal } \\
\text { health. }\end{array}$ \\
\hline $\begin{array}{l}\text { SFAs } \\
{[11]}\end{array}$ & Prospective & 264 & $75 \mathrm{y}$ & Dentate & & & & $\begin{array}{l}\text { Non-smokers in the highest } \\
\text { quartile of saturated fatty acids } \\
\text { intake had an increased risk for } \\
\text { periodontal disease events (ARR } \\
\quad=1.92 \text {, highest quartile). }\end{array}$ \\
\hline $\begin{array}{l}\text { DHA, } \\
\text { EPA, } \\
\text { LNA } \\
{[14]}\end{array}$ & $\begin{array}{l}\text { Cross- } \\
\text { sectional } \\
\text { study } \\
\text { NHANES } \\
\text { 1999-2004 }\end{array}$ & 9182 & $\geq 20 \mathrm{y}$ & Dentate & & & & $\begin{array}{l}\text { Those in the highest tertile of } \\
\text { DHA intake had lower odds of } \\
\text { periodontal disease (OR }=0.78 \text {, } \\
\text { highest tertile). NS associations } \\
\text { with either EPA or LNA acid. }\end{array}$ \\
\hline $\begin{array}{l}\text { DHA, } \\
\text { EPA } \\
{[15]}\end{array}$ & Prospective & 36 & $74 \mathrm{y}$ & Dentate & & & & $\begin{array}{l}\text { Those in the lowest tertile of } \\
\text { DHA intake had increased inci- } \\
\text { dence of periodontal disease } \\
\text { events (IRR }=1.49 \text {, lowest ter- } \\
\text { tile). NS associations with EPA. }\end{array}$ \\
\hline $\begin{array}{l}\text { Long chain n-3 } \\
\text { PUFA, } \gamma- \\
\text { linolenic acid } \\
{[12]}\end{array}$ & Pilot study & 30 & Adult & With periodontitis & $12 \mathrm{wk}$ & $3 \mathrm{~g}$ placebo & $\begin{array}{l}3 \mathrm{~g} \text { fish oil, or } \\
3 \mathrm{~g} \text { borage oil, or } \\
1.5 \mathrm{~g} \text { of each }\end{array}$ & $\begin{array}{l}\text { Improvement in probing depth } \\
\text { and gingival inflammation for } \\
\text { borage oil group, trend for fish } \\
\text { oil and combination groups. No } \\
\text { significant differences in plaque } \\
\text { index. }\end{array}$ \\
\hline $\begin{array}{c}\text { n-6 PUFA, } \\
\text { n-3 PUFA } \\
{[16]}\end{array}$ & Prospective & 235 & $75 \mathrm{y}$ & Dentate & & & & $\begin{array}{l}\text { Those in highest tertile of total } \mathrm{n} \text { - } \\
6 \text { PUFA compared to total n- } 3 \\
\text { PUFA intake were at a greater } \\
\text { risk of periodontal disease events } \\
\text { (ARR }=1.29 \text {, highest tertile). }\end{array}$ \\
\hline $\begin{array}{c}\text { Total CHO } \\
{[17]}\end{array}$ & $\begin{array}{l}\text { Single- } \\
\text { blind cross- } \\
\text { over study }\end{array}$ & 20 & $\begin{array}{l}\text { Young } \\
\text { adults }\end{array}$ & & $3 \mathrm{wk}$ & $\begin{array}{l}\text { Low sugar } \\
\text { diet }\end{array}$ & High sugar diet & $\begin{array}{l}\text { Higher bleeding scores in high } \\
\text { sugar group. No significant } \\
\text { differences in plaque score. }\end{array}$ \\
\hline $\begin{array}{c}\text { Dietary fibre } \\
\qquad[18]\end{array}$ & $\begin{array}{c}\text { Prospective } \\
\text { observation } \\
\text { study, } \\
\text { Health } \\
\text { Profession- } \\
\text { als Follow- } \\
\text { Up Study }\end{array}$ & 34160 & $40-75 y$ & $\begin{array}{l}\text { Excluded those } \\
\text { with periodontitis, } \\
\text { MI, stroke, diabe- } \\
\text { tes hyperchol- } \\
\text { esterolemia at } \\
\text { beginning of study }\end{array}$ & $12 \mathrm{y}$ & & & $\begin{array}{l}\text { Those in highest quintile of } \\
\text { whole grain intake were } 23 \% \text { less } \\
\text { likely to get periodontitis than } \\
\text { those in lowest quintile. Perio- } \\
\text { dontitis was not associated with } \\
\text { refined grain intake. Periodontal } \\
\text { risk was inversely related to } \\
\text { cereal fibre. }\end{array}$ \\
\hline \multicolumn{9}{|c|}{ Micronutrients with Potential Osteogenic Activity } \\
\hline $\begin{array}{c}\text { Calcium } \\
\text { [19] }\end{array}$ & $\begin{array}{l}\text { Longitudi- } \\
\text { nal study }\end{array}$ & 189 & $59 \mathrm{y}$ & $\begin{array}{l}\text { Healthy, dentate, } \\
\text { post-menopausal } \\
\text { women with } \\
\text { normal spine } \\
\text { density }\end{array}$ & $2 \mathrm{y}$ & $\begin{array}{c}\text { Placebo } \\
\text { taken daily }\end{array}$ & $\begin{array}{l}500 \mathrm{mg} \text { elemental } \\
\text { calcium either in } \\
\text { calcium citrate } \\
\text { malate or calcium } \\
\text { carbonate daily }\end{array}$ & $\begin{array}{l}\text { Greater proportion of non- } \\
\text { smoking placebo group lost teeth } \\
\text { than non-smoking supplemented } \\
\text { group. Those who lost teeth } \\
\text { during } 7 \mathrm{y} \text { follow up had greater } \\
\text { reduction of BMD at whole body, } \\
\text { femoral neck, and spine. For each } \\
1 \% / \mathrm{y} \text { decrement in BMD, there } \\
\text { was a higher relative risk of } \\
\text { losing tooth. }\end{array}$ \\
\hline
\end{tabular}


Table 2. contd....

\begin{tabular}{|c|c|c|c|c|c|c|c|c|}
\hline \multirow[t]{2}{*}{$\begin{array}{c}\text { Dietary } \\
\text { Component } \\
\text { [Reference] }\end{array}$} & \multirow[t]{2}{*}{$\begin{array}{l}\text { Study } \\
\text { Type }\end{array}$} & \multicolumn{3}{|c|}{ Characteristics of Participants } & \multirow[t]{2}{*}{$\begin{array}{l}\text { Intervention } \\
\text { Duration }\end{array}$} & \multicolumn{2}{|c|}{$\begin{array}{c}\text { Intervention/Comparison } \\
\text { Groups }\end{array}$} & \multirow[t]{2}{*}{ Outcomes } \\
\hline & & Number & Age & $\begin{array}{l}\text { Clinical } \\
\text { Condition }\end{array}$ & & Control & Treatment & \\
\hline $\begin{array}{c}\text { Calcium } \\
{[20]}\end{array}$ & $\begin{array}{l}\text { Random- } \\
\text { ized, clini- } \\
\text { cal study }\end{array}$ & 59 & & $\begin{array}{l}\text { With advanced } \\
\text { periodontal dis- } \\
\text { ease }\end{array}$ & $180 \mathrm{~d}$ & $\begin{array}{l}1 \mathrm{~g} \text { placebo } \\
\text { tablets daily }\end{array}$ & $\begin{array}{l}1 \mathrm{~g} \text { calcium tablet } \\
\text { daily }\end{array}$ & $\begin{array}{l}\text { No significant differences in } \\
\text { probing depth, gingival inflam- } \\
\text { mation or plaque score. }\end{array}$ \\
\hline $\begin{array}{c}\text { Calcium } \\
{[21]}\end{array}$ & $\begin{array}{l}\text { Cross- } \\
\text { sectional } \\
\text { study, } \\
\text { NHANES } \\
\text { III }\end{array}$ & & & & & & & $\begin{array}{l}\text { Association of lower calcium } \\
\text { intake with periodontal disease } \\
\text { for young males and females ( } 20 \text { - } \\
39 \text { y) and older males }(40-59 \mathrm{y}) \text {. } \\
\text { Dose response in females }(54 \% \\
\text { greater risk for lowest level of } \\
\text { intake }(<499 \mathrm{mg}), 27 \% \text { greater } \\
\text { risk for moderate intake }(500-799 \\
\mathrm{mg} \text { ) compared to those with } \\
\text { higher intakes }(>800 \mathrm{mg}) \text { ). Asso- } \\
\text { ciation between low total serum } \\
\text { calcium and periodontal disease } \\
\text { in younger females } 20-39 \text { y but } \\
\text { not for males or older females. }\end{array}$ \\
\hline $\begin{array}{l}\text { Calcium, } \\
\text { vitamin D } \\
{[22]}\end{array}$ & $\begin{array}{l}\text { Cross- } \\
\text { sectional } \\
\text { study }\end{array}$ & 228 & $\begin{array}{l}\text { Mean } \\
\text { age: } \\
63.6 \mathrm{y}\end{array}$ & $\begin{array}{l}\text { With periodontal } \\
\text { disease }\end{array}$ & & & & $\begin{array}{l}\text { Only } 7 \% \text { of participants met } \\
\text { RDAs of calcium and vitamin D. } \\
66 \% \text { did not take oral supple- } \\
\text { ments. More males than females } \\
\text { who did not take calcium sup- } \\
\text { plements. }\end{array}$ \\
\hline $\begin{array}{c}\text { Dairy intake } \\
\text { [23] }\end{array}$ & $\begin{array}{l}\text { Cross- } \\
\text { sectional } \\
\text { study, } \\
\text { NHANES } \\
\text { III }\end{array}$ & 12764 & & & & & & $\begin{array}{l}\text { Prevalence of periodontitis was } \\
41 \% \text { lower for people in highest } \\
\text { quintile of dairy intake than those } \\
\text { in lowest quintile. }\end{array}$ \\
\hline $\begin{array}{c}\text { Calcium, } \\
\text { vitamin D } \\
\text { [7] }\end{array}$ & $\begin{array}{l}\text { Cross- } \\
\text { sectional } \\
\text { study }\end{array}$ & 51 & & $\begin{array}{c}\text { With } 2 \text { or more } \\
\text { interproximal sites } \\
\text { with } 3 \mathrm{~mm} \text { clinical } \\
\text { attachment loss or } \\
\text { more }\end{array}$ & & $\begin{array}{l}\text { Not taking } \\
\text { supplements }\end{array}$ & $\begin{array}{l}1000 \mathrm{mg} \text { calcium } \\
\text { and } 400 \text { IU vita- } \\
\text { min D daily }\end{array}$ & $\begin{array}{l}\text { Trend in shallower probing } \\
\text { depths, fewer bleeding sites, } \\
\text { lower gingival index values, } \\
\text { fewer furcation involvements, } \\
\text { less attachment loss, and less } \\
\text { alveolar crest height loss but } \\
\text { results were not significant. }\end{array}$ \\
\hline $\begin{array}{c}\text { Calcium, } \\
\text { vitamin D } \\
{[8]}\end{array}$ & $\begin{array}{l}\text { Double- } \\
\text { blind, } \\
\text { random- } \\
\text { ized, pla- } \\
\text { cebo- } \\
\text { controlled } \\
\text { study }\end{array}$ & 145 & $>65 \mathrm{y}$ & Healthy & $3 y$ & Placebo pills & $\begin{array}{l}500 \mathrm{mg} \text { calcium } \\
\text { and } 700 \text { IU vita- } \\
\text { min D daily }\end{array}$ & $\begin{array}{l}\text { Lower odds of tooth loss were } \\
\text { associated with supplement status } \\
\text { during study period, and total } \\
\text { calcium intake during follow up. } \\
\text { NS differences in probing depths. }\end{array}$ \\
\hline $\begin{array}{l}\text { Vitamin D } \\
\text { [19] }\end{array}$ & $\begin{array}{l}\text { Longitudi- } \\
\text { nal study }\end{array}$ & 189 & $59 \mathrm{y}$ & $\begin{array}{l}\text { Healthy, dentate, } \\
\text { post-menopausal, } \\
\text { with normal spine } \\
\text { density }\end{array}$ & $1 \mathrm{y}$ & $\begin{array}{l}\text { Placebo with } \\
377 \mathrm{mg} \\
\text { calcium daily }\end{array}$ & $\begin{array}{l}400 \text { IU vitamin D } \\
\text { with } 377 \mathrm{mg} \\
\text { calcium daily }\end{array}$ & $\begin{array}{l}\text { No effect on tooth loss. Those } \\
\text { who lost teeth during } 7 \text { y follow- } \\
\text { up had greater reduction of BMD } \\
\text { at whole body, femoral neck, and } \\
\text { spine. For each } 1 \% \text { per y decre- } \\
\text { ment in BMD, higher relative } \\
\text { risk of losing tooth. }\end{array}$ \\
\hline $\begin{array}{c}\text { Vitamin D } \\
\text { [19] }\end{array}$ & $\begin{array}{l}\text { Longitudi- } \\
\text { nal study }\end{array}$ & 189 & $59 \mathrm{y}$ & $\begin{array}{l}\text { Healthy, dentate, } \\
\text { post-menopausal, } \\
\text { with normal spine } \\
\text { density }\end{array}$ & $2 y$ & $\begin{array}{c}100 \mathrm{IU} \\
\text { vitamin } \mathrm{D} \\
\text { with } 500 \mathrm{mg} \\
\text { calcium daily }\end{array}$ & $\begin{array}{l}700 \text { IU vitamin D } \\
\text { with } 500 \mathrm{mg} \\
\text { calcium daily }\end{array}$ & $\begin{array}{l}\text { No effect on tooth loss. Those } \\
\text { who lost teeth during } 7 \text { y follow- } \\
\text { up had greater reduction of BMD } \\
\text { at whole body, femoral neck, and } \\
\text { spine. For each } 1 \% / y \text { decrement } \\
\text { in BMD, higher relative risk of } \\
\text { losing tooth. }\end{array}$ \\
\hline $\begin{array}{c}\text { Vitamin D } \\
\text { [9] }\end{array}$ & $\begin{array}{l}\text { Cross- } \\
\text { sectional } \\
\text { study, } \\
\text { NHANES } \\
\text { III }\end{array}$ & 3781 & $>50 \mathrm{y}$ & & & & & $\begin{array}{l}\text { Inverse relationship between } \\
\text { attachment loss and serum } \\
25(\mathrm{OH}) \mathrm{D}\end{array}$ \\
\hline
\end{tabular}


Table 2. contd....

\begin{tabular}{|c|c|c|c|c|c|c|c|c|}
\hline \multirow[t]{2}{*}{$\begin{array}{l}\text { Dietary } \\
\text { Component } \\
\text { [Reference] }\end{array}$} & \multirow[t]{2}{*}{$\begin{array}{l}\text { Study } \\
\text { Type }\end{array}$} & \multicolumn{3}{|c|}{ Characteristics of Participants } & \multirow[t]{2}{*}{$\begin{array}{l}\text { Intervention } \\
\text { Duration }\end{array}$} & \multicolumn{2}{|c|}{$\begin{array}{c}\text { Intervention/Comparison } \\
\text { Groups }\end{array}$} & \multirow[t]{2}{*}{ Outcomes } \\
\hline & & Number & Age & $\begin{array}{l}\text { Clinical } \\
\text { Condition }\end{array}$ & & Control & Treatment & \\
\hline $\begin{array}{l}\text { Vitamin D } \\
\text { [24] }\end{array}$ & $\begin{array}{l}\text { Cross- } \\
\text { sectional } \\
\text { study, } \\
\text { NHANES } \\
\text { III }\end{array}$ & 6700 & $>13$ y & Never smokers & & & & $\begin{array}{l}\text { Participants in highest quintile of } \\
\text { serum } 25(\mathrm{OH}) \mathrm{D} \text { were } 20 \% \text { less } \\
\text { likely to bleed on probing. }\end{array}$ \\
\hline $\begin{array}{l}\text { Magnesium } \\
{[25]}\end{array}$ & $\begin{array}{l}\text { Cross- } \\
\text { sectional } \\
\text { study }\end{array}$ & 2931 & $>40 \mathrm{y}$ & $\begin{array}{c}33 \% \text { had hypo- } \\
\text { magnesemia }\end{array}$ & & & & $\begin{array}{l}\text { Inverse relationships between } \\
\text { serum } \mathrm{Mg} \text { and lower probing } \\
\text { depth and attachment loss }\end{array}$ \\
\hline $\begin{array}{c}\text { Fluoride } \\
\text { [26] }\end{array}$ & $\begin{array}{l}\text { Double- } \\
\text { blind, } \\
\text { random- } \\
\text { ized, paral- } \\
\text { lel study }\end{array}$ & 70 & $\begin{array}{l}>18 \mathrm{y} \\
(\text { mean } \\
\text { age of } \\
30 \mathrm{y})\end{array}$ & $\begin{array}{l}\text { Generalized } \\
\text { gingival inflam- } \\
\text { mation with some } \\
\text { dentinal sensitiv- } \\
\text { ity, no acute } \\
\text { gingival or perio- } \\
\text { dontal condition }\end{array}$ & $4 \mathrm{wk}$ & $\begin{array}{l}\text { Placebo(de- } \\
\text { ionized } \\
\text { water) }\end{array}$ & $\begin{array}{c}\text { Natural mineral } \\
\text { dietary supple- } \\
\text { ment with } 3.6 \mathrm{mg} \\
\mathrm{I}^{-1} \text { of } \mathrm{F} \text { and other } \\
\text { minerals in trace } \\
\text { amounts }(\mathrm{Si}, \\
\mathrm{HCO}, \mathrm{Na}, \mathrm{Cl}, \mathrm{K}, \\
\text { Ca etc) }\end{array}$ & $\begin{array}{l}\text { No significant differences in } \\
\text { gingival inflammation. }\end{array}$ \\
\hline \multicolumn{9}{|c|}{ Micronutrients and Food Bioactives with Potential Antioxidant Activity } \\
\hline $\begin{array}{l}\text { Vitamin C } \\
{[30]}\end{array}$ & $\begin{array}{l}\text { Cross- } \\
\text { sectional } \\
\text { study, } \\
\text { NHANES } \\
\text { III }\end{array}$ & 12419 & $>20 \mathrm{y}$ & & & & & $\begin{array}{c}\text { Reduction of dietary vitamin } \mathrm{C} \\
\text { was related with attachment level } \\
\text { of }>=1.5 \mathrm{~mm} \text { in overall popula- } \\
\text { tion. Higher risk for current } \\
\text { smokers and former smokers who } \\
\text { took less dietary vitamin C. Dose } \\
\text { response relationship exists } \\
(\mathrm{OR}=1.3,0-29 \mathrm{mg} \text { vitamin } \mathrm{C} \\
\mathrm{OR}=1.16,100-179 \mathrm{mg} \text { vitamin } \mathrm{C} \\
\mathrm{OR}=1,180 \mathrm{mg}+\text { vitamin } \mathrm{C})\end{array}$ \\
\hline $\begin{array}{l}\text { Vitamin C } \\
{[31]}\end{array}$ & $\begin{array}{l}\text { Cross- } \\
\text { sectional } \\
\text { study }\end{array}$ & 413 & $70 \mathrm{y}$ & & & & & $\begin{array}{l}\text { Inverse relationship between } \\
\text { serum vitamin } C \text { and clinical } \\
\text { attachment loss. }\end{array}$ \\
\hline $\begin{array}{l}\text { Vitamin } C \\
{[32]}\end{array}$ & $\begin{array}{l}\text { Case- } \\
\text { matched } \\
\text { study }\end{array}$ & 10 & $>30 \mathrm{y}$ & $\begin{array}{l}\text { Non-deficient in } \\
\text { vitamin C, with } \\
\text { gingivitis }\end{array}$ & $6 \mathrm{wk}$ & $\begin{array}{l}\text { Placebo, } 4 \\
\text { pills daily }\end{array}$ & $\begin{array}{l}250 \mathrm{mg} \text { ascorbic } \\
\text { acid in each pill, } \\
4 \text { pills daily }\end{array}$ & $\begin{array}{c}\text { No significant differences in } \\
\text { probing depth, attachment level, } \\
\text { gingival inflammation, and } \\
\text { plaque level. }\end{array}$ \\
\hline $\begin{array}{c}\text { Vitamin } C \\
\text { [33] }\end{array}$ & $\begin{array}{l}\text { Single- } \\
\text { blind study }\end{array}$ & 30 & $>20 \mathrm{y}$ & $\begin{array}{l}\geq 12 \text { remaining } \\
\text { teeth, ability to } \\
\text { develop calculus, } \\
\text { otherwise healthy }\end{array}$ & $3 \mathrm{mo}$ & $\begin{array}{c}\text { Vitamin C } \\
\text { and sugar } \\
\text { free chewing } \\
\text { gum, } 5 \text { times } \\
\quad \text { daily } \\
\quad \text { or } \\
\text { No chewing } \\
\text { gum }\end{array}$ & $\begin{array}{l}60 \mathrm{mg} \text { vitamin } \mathrm{C} \\
\text { in each sugar free } \\
\text { chewing gum, } 5 \\
\text { times daily }\end{array}$ & $\begin{array}{l}\text { Lower bleeding scores in vitamin } \\
\text { C gum chewers than non gum } \\
\text { chewers. Lower visible plaque } \\
\text { index in gum chewers than non } \\
\text { gum chewers. }\end{array}$ \\
\hline $\begin{array}{c}\text { Vitamin C } \\
{[34]}\end{array}$ & $\begin{array}{l}\text { Longitudi- } \\
\text { nal, single- } \\
\text { blinded } \\
\text { randomized } \\
\text { study }\end{array}$ & 80 & $22-75 y$ & $\begin{array}{l}\text { With chronic } \\
\text { periodontitis, } \\
\mathrm{n}=58 \\
\text { These subjects } \\
\text { were divided into } \\
\text { a test group } \\
(\mathrm{n}=38) \text { and dis- } \\
\text { eased control } \\
\text { group }(\mathrm{n}=20) \\
22 \text { healthy sub- } \\
\text { jects were also } \\
\text { studied. }\end{array}$ & $2 \mathrm{wk}$ & $\begin{array}{l}\text { No consump- } \\
\text { tion of grape- } \\
\text { fruits for } \\
\text { patients with } \\
\text { chronic } \\
\text { perio-dontitis } \\
\quad \text { or } \\
\text { No consump- } \\
\text { tion of grape- } \\
\text { fruit for } \\
\text { healthy } \\
\text { subjects }\end{array}$ & $\begin{array}{l}\text { Two grapefruits } \\
\text { daily for patients } \\
\text { with chronic } \\
\text { periodontitis }\end{array}$ & $\begin{array}{l}\text { Lower sulcus bleeding index. No } \\
\text { effect on probing depth and } \\
\text { plaque index. }\end{array}$ \\
\hline $\begin{array}{l}\text { Vitamin E } \\
\text { [29] }\end{array}$ & $\begin{array}{l}\text { Random- } \\
\text { ized study }\end{array}$ & 409 & $55-74 y$ & Smokers & $3 \mathrm{yrs}$ & $\begin{array}{c}\text { ASA } \\
\text { or } \\
\text { Neither } \\
\text { vitamin E } \\
\text { nor ASA }\end{array}$ & $\begin{array}{l}50 \mathrm{mg} \text { vitamin } \mathrm{E} \\
\text { supplement-ation } \\
\text { daily } \\
\text { or } \\
\text { Both vitamin E } \\
\text { and ASA daily }\end{array}$ & $\begin{array}{l}\text { Gingival inflammation was more } \\
\text { common in vitamin E supple- } \\
\text { mented group than non receivers. } \\
\text { Highest risk in group that re- } \\
\text { ceived both. Higher prevalence } \\
\text { of dental plaque in vitamin E } \\
\text { supplemented group. }\end{array}$ \\
\hline
\end{tabular}


Table 2. contd....

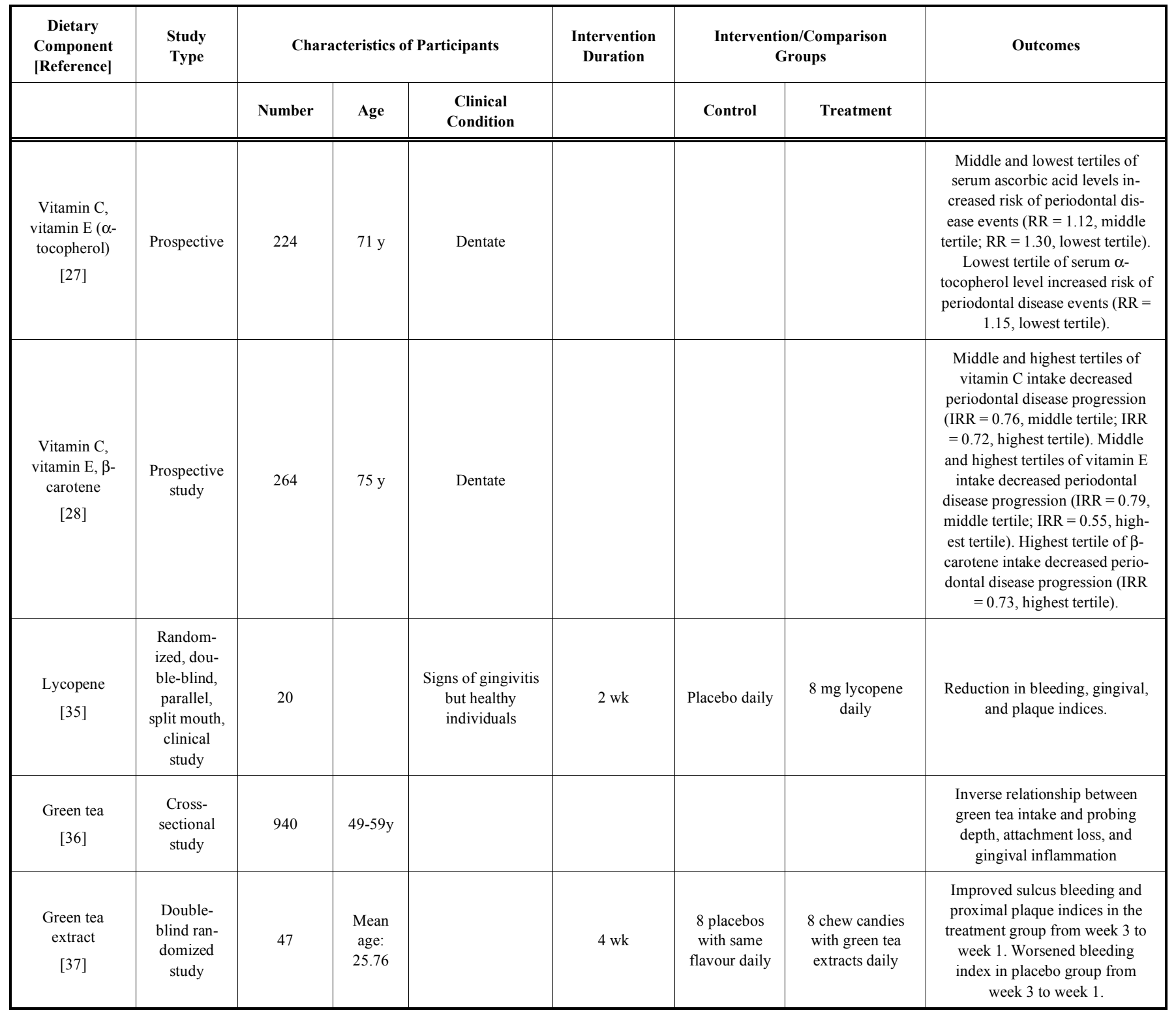

ARR, adjusted relative risk; BMD, bone mineral density; CHO, carbohydrate; DHA, docosahexaenoic acid; EPA, eicosapentaenoic acid; IRR, incidence rate ratio; LNA, linolenic acid; OR, odds ratio; PDR, probing depth reduction; PUFA, polyunsaturated fatty acid; RLBG, radiographic linear bone gain; RR, relative risk; ROS, reactive oxygen species; SFA, saturated fatty acid.

energy metabolism and thus may facilitate healing through these activities. Vitamin D has favorable effects by having direct positive effects on bone metabolism as well as immunomodulatory activity. As discussed later in this review, higher serum $25(\mathrm{OH}) \mathrm{D}$ levels are associated with less periodontal attachment loss [5, 7-9] and these studies suggest an immunomodulatory role for vitamin D.

\section{ASSOCIATIONS BETWEEN PERIODONTAL HEALTH AND DIETARY COMPONENTS WITH ANTI-INFLAMMATORY, OSTEOGENIC OR ANTI- OXIDANT ACTIVITY}

Because of the paucity of intervention studies examining diet and healing after an oral surgical procedure, a review of studies that examined the association between dietary components and periodontal health in adults, both healthy par- ticipants and those with varying degrees of periodontitis, is useful to more fully understand the role of diet in periodontal health (Table 2). In Table $\mathbf{2}$, these studies are grouped by category - macronutrients or micronutrients - and within these categories, by their potential biological effect such as anti-inflammatory activity, osteogenic activity or antioxidant activity.

A prospective, randomized study was conducted with 148 families to determine whether educating the parents to adopt anti-atherogenic diets would influence any health outcomes including periodontal status [10]. After 29 months, there were no differences in periodontal health of parents who consumed diets with a high ratio of polyunsaturated fatty acid (PUFA) to saturated fat acid (SFA), suggesting that fat quality has no effect on the adult periodontium. However, a recent prospective study in older individuals, i.e. 
75 years of age and older, suggests that a diet high in SFA may increase the risk of periodontal disease in non-smokers [11]. It is suspected that a diet high in SFA produces a proinflammatory environment that enables the initiation and progression of periodontal disease. In contrast, although smoking is a well-established risk factor for developing periodontal disease, there was no relationship between SFA intake and periodontal disease among smokers. It is possible that a relationship was masked because the negative effects of smoking to periodontium may dominate and mask the proinflammatory effects of SFA.

A clinical trial showed positive effects of borage oil [12], a rich source of gamma linolenic acid (GLA). The known anti-inflammatory properties of GLA led to the postulation that they may attenuate inflammation in many different disease states [13]. Among patients with periodontitis, significant improvements from baseline were observed with three grams of borage oil supplementation but not with three grams of fish oil per day [12]. Further study is needed to understand how the type and ratio of specific fatty acids impacts periodontal health. Dose-response studies with specific fatty acids are also warranted, particularly as data from the National Health and Nutritional Examination Survey (NHANES), suggests that diets that are higher in docosahexaenoic acid (DHA), a n-3 PUFA, are associated with decreased odds of periodontal disease [14]. This association has recently been substantiated by a prospective study in which the incidence of periodontal disease was 1.5 times greater in those with the lowest DHA intake compared to those with the highest DHA intake [15]. At present, the association between DHA intake and periodontal disease is best explained by the known anti-inflammatory effect of omega-3 fatty acids. Also, a prospective study in older adults has identified that a diet containing a higher ratio of n-6 to $n-3$ PUFAs was associated with significantly more periodontal disease events [16]. Thus, findings to date suggest that type of dietary fat may modulate periodontal health and further investigation is warranted.

Dietary carbohydrates may also modulate periodontal health. Results from a single crossover study demonstrated that a high sugar diet contributed to higher bleeding scores as compared to a low sugar diet in young adults with no differences in plaque score [17]. In the Health Professionals Follow Up Study, 34,160 participants aged 40-75 years were followed for 12 years and participants with the highest quintile of whole grain intake were $23 \%$ less likely to get periodontitis than those with the lowest quintile [18]. This suggests that fibre may be associated with the initiation of the disease, at least in this population group of middle aged and older adults. These findings suggest that a diet low in sugar and high in fibre may be helpful in moderating inflammation in the periodontium [18].

In contrast to macronutrients, more studies have investigated the role of micronutrients, particularly calcium and vitamin $\mathrm{D}$ in periodontal health [7-9, 19-24]. This is likely because supplementation with calcium and vitamin $\mathrm{D}$ is common among older individuals, and has proven beneficial for overall bone health including maintenance of bone mineral density and protection against fragility fractures. Because the periodontium presents unique structural and func- tional features apart from bone it is important to explore if and how calcium and vitamin D affect the periodontal state in an age group that is more vulnerable to periodontitis.

In a group of healthy, dentate, postmenopausal female nonsmokers with normal bone mineral density (BMD), a daily supplementation of $500 \mathrm{mg}$ of calcium provided as calcium citrate malate or calcium carbonate taken for two years lowered the risk of tooth loss as compared to the nonsmoking placebo group [19]. Of note is the fact that half of the participants enrolled in this study had dietary calcium intakes of less than $400 \mathrm{mg} /$ day, and thus had intakes that were substantially lower than the daily recommendation of $1200 \mathrm{mg} /$ day for adults over 50 years of age. The study also demonstrated a relationship between tooth retention and loss of BMD at multiple skeletal sites (spine, hip, wrist) [19]. Individuals who lost one or more teeth during the seven year follow up period also experienced a greater reduction of BMD at whole body, femoral neck, and spine sites [19]. Conversely, for each percent per year decrement in BMD, a higher relative risk of tooth loss was observed [19]. As tooth loss is dependent on alveolar bone integrity, it is likely that the deterioration of alveolar bone in age-related bone loss is coupled with the overall decrease in BMD, leading to poor tooth retention. Calcium supplementation may help maintain alveolar bone health and thus tooth retention; this may be especially important to those who have a low calcium intake, below recommended levels.

While dietary calcium may slow alveolar bone loss in healthy aging subjects, a randomized clinical trial had reported that in patients with advanced periodontitis, there were no differences in probing depth, gingival inflammation, and plaque levels between those who were administered $1 \mathrm{~g}$ calcium tablets daily for 180 days or those who had taken placebo tablets for the same duration [20]. Because the recommended intake for calcium was $800 \mathrm{mg} /$ day when the study was conducted, the authors classified participants as having intakes above or below $800 \mathrm{mg}$ calcium/day, and evenly randomized participants with these different intakes among the treatment and placebo group. Comparing the participants in the test group with different intakes yielded no significant differences. In contrast, those in the placebo group with a calcium intake greater than $800 \mathrm{mg}$ calcium/day had a lower probing depth as well as a higher gingival index than those with an intake lower than $800 \mathrm{mg}$ calcium/day. The conflicting results might arise from the fact that the intakes of most participants were around $800 \mathrm{mg}$ calcium/day, limiting the difference in characteristics between the two groups. Since the etiology of periodontal disease involves heightened inflammatory processes, it is possible that supplemental calcium may not be directly involved in the attenuation of inflammation. Since tooth retention was not assessed in this study, the possible benefit of supplemental calcium in preserving alveolar bone density was not tested.

Another study also suggests that low calcium intake may be detrimental to periodontal health. Data from cross-sectional studies from the NHANES III showed that a lower calcium intake was associated with periodontal disease, as determined by clinical attachment loss, for young males and females (2039 y) and older males (40-59 y) [21]. The association with periodontal disease risk was dose-dependent in females. Fe- 
males who had daily calcium intakes of less than $500 \mathrm{mg}$ had a $54 \%$ greater risk of periodontal disease than those who took more than $800 \mathrm{mg}$. Females who consumed 500 to $799 \mathrm{mg}$ of calcium daily had a $27 \%$ greater risk [21]. It is known that calcium intakes are often substantially lower than recommended levels, and a recent cross-sectional study conducted on patients with periodontitis confirmed this finding [22]. Only $7 \%$ of the participants, with an average age of 64 years, consumed calcium and vitamin D at the recommended levels [22].

Studies investigating the combined effects of calcium and vitamin D supplementation have, in general, not shown a significant relationship with promotion of periodontal health $[7,8,19]$. However, dairy intake is associated with a significantly lower prevalence of periodontitis for individuals in the highest quintile of dairy intake than those in the lowest quintile [23]. In patients with at least $3 \mathrm{~mm}$ of clinical attachment loss, there were no significant differences in probing depths, number of bleeding sites, gingival index values, number of furcation involvements, attachment loss, and alveolar crest height loss among subjects taking more than $1000 \mathrm{mg}$ of calcium and $400 \mathrm{IU}$ of vitamin D daily [7]. However, trends for improvements in these outcomes were noted and suggest that further study is warranted. No significant differences in probing depths were found in healthy subjects over 65 years of age who took a daily supplement of $500 \mathrm{mg}$ of calcium and 700 IU of vitamin D for three years, although the odds of tooth loss were associated with supplement status [8].

One study reports no effect of providing calcium and vitamin D supplements on tooth loss [19]. In this study, healthy postmenopausal women received either 400 IU of vitamin D or a placebo daily for a year, and 700 IU of vitamin D or 100 IU of vitamin D daily for two years [19]. Subjects, half of whom had a daily intake of calcium less than $400 \mathrm{mg}$ and half had a daily intake between 400-650 mg, were given $377 \mathrm{mg}$ and $500 \mathrm{mg}$ of calcium supplements respectively [19]. The fact that an inclusion criterion was a normal spine BMD may explain why tooth retention was not altered with supplementation.

Two cross-sectional studies from the NHANES III data showed a relationship between vitamin $\mathrm{D}$ and periodontal health. In 3781 adults over 50 years of age, there was an inverse relationship between attachment loss and serum $25(\mathrm{OH}) \mathrm{D}[9]$. In this study, the dietary calcium intake for both males and females were lower in the participants over age 50 than those from 20 to 49 years of age, and the average intakes for the four groups were below the recommended 1000-1200 $\mathrm{mg}$ /day [9]; the lower intake of calcium may have lessened the effect of vitamin D. Similarly, it was shown that of 6700 never-smokers with the highest quintile of serum $25(\mathrm{OH}) \mathrm{D}$ (average serum concentration of $106 \mathrm{nmol} 25(\mathrm{OH}) \mathrm{D} / \mathrm{L}$ ), were $20 \%$ less likely to bleed on probing than those in the lowest quintile with an average serum concentration of $31 \mathrm{nmol}$ $25(\mathrm{OH}) \mathrm{D} / \mathrm{L}$ [24]. Although these studies do not directly link supplementation with vitamin D to periodontal status, taking dietary supplements is a viable method to increase serum levels of vitamin $\mathrm{D}$ in a population that has calcium and vitamin $\mathrm{D}$ intakes below the recommendation.

Studies that focused on other minerals are very limited. Inverse relationships between serum magnesium and probing depth and attachment loss were reported in a cross-sectional study that included 2931 adults [25]. In adults with generalized gingival inflammation, supplementing water with fluoride and other trace minerals yielded no differences in gingival inflammation compared with de-ionized water [26].

In a prospective study, older individuals ( 75 years of age and older) in the lowest tertile of serum vitamin $E(\alpha-$ tocopherol) were associated with more periodontal disease, while those in the middle and highest tertiles of vitamin $\mathrm{E}$ intake were associated with lower periodontal disease [27]. Moreover, individuals with the highest intakes of fruits and vegetables had a lower number of teeth with periodontal disease progression [28]. Although supplement use was not measured in this study, it is interesting that a relationship between higher dietary vitamin $\mathrm{E}$ intake and lower periodontal disease was observed when examining dietary levels alone. Another study showed that individuals taking vitamin E (50 $\mathrm{mg}$ vitamin D/day) or acetylsalicylic acid (ASA, 100-3200 $\mathrm{mg}$ /day) had enhanced gingival bleeding compared to individuals taking neither vitamin E or ASA alone if they had dental plaque on $40 \%$ of measured surfaces [29]. Not surprisingly, a higher amount of dental plaque was associated with greater gingival bleeding and the enhanced bleeding with the combination of vitamin E and ASA was observed in individuals with greater but not less dental plaque [29]. The authors of the study attributed the outcome to an enhanced inhibition of blood clotting by vitamin E [29]. Further understanding of how vitamin E supplements modulate blood clot formation one of the first steps in healing - is needed.

Low intakes of vitamin $C$ [30] and low serum vitamin C $[27,31]$ are associated with greater clinical attachment loss in adults. Moreover, a high intake of vitamin $\mathrm{C}$, at a level that meets or is in excess of the current dietary recommended intakes for vitamin $\mathrm{C}$ in North America, is associated with a decrease in periodontal disease progression [28]. A doseresponse relationship between the risk of clinical attachment loss and dietary vitamin $\mathrm{C}$ has been demonstrated. Subjects with a low intake of vitamin $\mathrm{C}(<29 \mathrm{mg} /$ day $)$ were 1.3 times more likely to have clinical attachment loss than those with more than $180 \mathrm{mg}$ of vitamin $\mathrm{C}$ [30]. Current and former smokers were especially placed at a greater risk [30]. Although low levels of vitamin $\mathrm{C}$ intake may be indicative of poorer periodontal health, intake ceases to be influential if the individual is consuming vitamin $\mathrm{C}$ at recommended levels: $90 \mathrm{mg} / \mathrm{d}$ for men and $75 \mathrm{mg} / \mathrm{d}$ for women. For example, subjects who consumed vitamin $\mathrm{C}$ at recommended levels did not have improved outcomes (probing depth, clinical attachment levels, gingival inflammation, plaque level) after receiving $1000 \mathrm{mg}$ of ascorbic acid for six months [32]. Another study showed that after chewing sugar free gum, each piece containing $60 \mathrm{mg}$ of vitamin $\mathrm{C}$, five times daily for three months, participants had lower bleeding scores than non-gum chewers, while sugar free gum without vitamin $\mathrm{C}$ had no effect [33]. Another study examined if grapefruit, a rich source of vitamin $\mathrm{C}$, modulated chronic periodontitis. Patients with chronic periodontitis who consumed two grapefruits daily for two weeks had a lower sulcus bleeding index than those who did not, although there were no differences in probing depth and plaque index [34]. Although it may be difficult to sustain a grapefruit eating habit for a prolonged period of time, eating a variety of fruits and vegeta- 
Table 3. Dietary Considerations Prior to Surgical Procedures

\begin{tabular}{|c|c|c|}
\hline Points to Consider & Reasoning & Approach \\
\hline \hline Current nutritional status & $\begin{array}{c}\text { Poor nutrition before surgery may not be ade- } \\
\text { quate for the increased nutritional needs after } \\
\text { surgery and may predispose patients to pro- } \\
\text { longed healing }\end{array}$ & $\begin{array}{c}\text { Assess food intake and advise the patient to meet the Dietary Rec- } \\
\text { ommended Intakes (DRI) by following Canada's Food Guide } \\
\text { Consider multivitamins or other supplements, keeping in mind the } \\
\text { Upper Tolerable Limit (UL) }\end{array}$ \\
\hline Current food consumption habits & $\begin{array}{c}\text { Food preferences may affect adherence to food } \\
\text { suggestions post-surgery }\end{array}$ & $\begin{array}{c}\text { Assess food preferences through food records } \\
\text { Be realistic about patient's compliance and adapt the food sugges- } \\
\text { tions to their preferences as much as possible }\end{array}$ \\
\hline
\end{tabular}

bles that are rich in vitamins offers an alternative to supplementation. The negative impact of having low levels of vitamin $\mathrm{C}$ is especially important to smokers and those who are undernourished. It is important to note that in addition to being a rich source of vitamin $\mathrm{C}$, grapefruit is also a source of flavonoids and other bioactives that may have a role in promoting periodontal health.

A few studies have reported the effects of other dietary components or foods with potential antioxidant activity such as $\beta$-carotene, lycopene, and green tea and its extract $[28,35$ 37]. In a prospective study, those consuming the greatest amounts of $\beta$-carotene in their diet were associated with a $27 \%$ reduction in the risk of periodontal disease progression [28]. Reductions in bleeding, gingival indices and plaque indices were recorded in healthy patients showing signs of gingivitis after taking $8 \mathrm{mg}$ of lycopene daily for two weeks compared to those who took the placebo [35]. Green tea demonstrated similarly positive results $[36,37]$. There was an inverse relationship between green tea intake and probing depth, clinical attachment loss, and bleeding on probing. This may have been due to the inhibition of periodontal pathogenic growth and production of virulence factors by green tea polyphenols as shown by in vitro studies [36]. Chewing on eight pieces of candy with green tea extract daily resulted in improved sulcus bleeding and proximal plaque indices from week one to week three, while these values worsened for those who received placebo [37].

In summary, findings from the studies discussed can be categorized according to their biological activities including anti-inflammatory, osteogenic and antioxidant mechanisms. More specifically, higher intakes of DHA and vitamin D through food sources or supplements, as well as a lower ratio of n-6 to n-3 fatty acids, lower carbohydrate and higher fibre intakes may be beneficial for healing due to antiinflammatory effects. Meeting dietary requirements for calcium and use of vitamin D supplements may benefit healing through osteogenic activity. The relationship among antioxidant nutrients such as vitamin $E$ and $\beta$-carotene and healing are complex and require further investigation while there is strong evidence that lower intakes of vitamin $\mathrm{C}$ are harmful to periodontal health. Other foods or food components with antioxidant activity such as green tea and lycopene have shown potential to improve periodontal health.

\section{DIETARY CONSIDERATIONS PRIOR TO AND POST-SURGICAL PROCEDURES}

Although studies to date are limited in number and in scope, they suggest that a healthful diet has the potential to accelerate oral wound healing and improve treatment outcome and patient satisfaction. In particular, there is some evidence that a wide variety of dietary components - including macronutrients and micronutrients - are integral for optimal periodontal health as well as healing after periodontal procedures. Prospective studies as well as intervention studies that assess multiple dietary components and overall dietary patterns in relation to wound healing are needed before detailed dietary strategies can be prescribed for patients undergoing complex periodontal procedures. Nonetheless, because of the known role of a balanced diet for overall health it is prudent to consider, prior to surgical procedures, the current nutritional status of a patient, as well as their current food consumption patterns (Table 3). Specific dietary components such as calcium, vitamin D, long chain PUFA are quite often consumed at levels below the recommended intakes; thus emphasizing the importance of increasing intakes in such individuals. It is also important that a patient does not consume individual nutrients in excessive amounts that may cause harm. In North America, the Dietary Reference Intakes (DRIs) are a comprehensive set of reference values for nutrients. These DRIs can assist with the assessment and planning of diets for healthy populations, and are based on gender and life stage. Moreover, the DRIs also provide guidance regarding the highest level of a nutrient that will likely pose no adverse health risk. Consideration of a patient's current food consumption habits is also warranted prior to a periodontal procedure. Food consumption habits can be extremely difficult for a patient to change, and thus it is important when providing dietary advice that it will be realistic for a patient to actually consume the foods advised. Individualized diet planning may be warranted, particularly for the patient who consumes foods that are not typical of the majority of patients. Ultimately, it is essential to have a realistic perspective regarding whether a patient will be able to adhere to the dietary advice given their food preferences. A helpful way to determine a patient's potential success in complying with dietary advice pre- and post-surgery may be obtained through use of a written dietary record of foods consumed in advance of the scheduled procedure.

Dietary considerations post-surgical procedures are also important to optimize healing (Table 4). Patients should be advised to take a multivitamin during the healing phase, particularly during the early phase of healing, to help ensure a patient is meeting the recommended levels of nutrients. However, it is important to recognize that the DRIs are established for healthy populations without preexisting disease and that higher levels of specific nutrients may be beneficial for healing. As discussed in this review, this is an area that 
Table 4. Dietary Considerations Post-Surgical Procedures

\begin{tabular}{|c|c|c|}
\hline Points to Consider & Reasoning & Approach \\
\hline Nutrition & $\begin{array}{l}\text { Wound healing may require higher } \\
\text { intakes of some foods } \\
\text { Maintaining a balanced intake of nutri- } \\
\text { ents by using supplements as needed, } \\
\text { i.e., Vitamin C may promote healing }\end{array}$ & $\begin{array}{c}\text { Educate the patient on the importance and the way to achieve the DRI } \\
\text { Consider multivitamins or other supplements, keeping in mind the Upper Tol- } \\
\text { erable Limit (UL) } \\
\text { Promote intake of nutrients that may accelerate healing } \\
\text { Consider pre-existing medical conditions (e.g. diabetes) and interactions with } \\
\text { medications } \\
\text { Offer referrals for nutritional counseling }\end{array}$ \\
\hline Ease of intake & $\begin{array}{l}\text { The consumption of food should be as } \\
\text { painless as possible without damaging } \\
\text { or aggravating the wound site }\end{array}$ & $\begin{array}{l}\text { Educate the patient to evaluate the texture, consistency, and temperature of } \\
\text { food that is appropriate to their stage in healing (i.e., Crunchy foods should be } \\
\text { avoided; foods such as cooked potatoes can be crushed or mashed to varying } \\
\text { degrees; chilled or cold foods may be more soothing to the wound site) } \\
\text { Consider different food processing methods to maintain proper nutrition and } \\
\text { introduce variety to keep the patient motivated } \\
\text { Encourage the patient to chew slowly and carefully } \\
\text { Consider medications that may modulate food intake }\end{array}$ \\
\hline Palatability & $\begin{array}{l}\text { Food that is appetizing encourages } \\
\text { lasting healthy eating habits and an } \\
\text { adequate intake of nutrients to promote } \\
\text { wound healing }\end{array}$ & $\begin{array}{l}\text { Consider texture or consistency, odor, color, temperature and taste of food } \\
\text { Consider the patient's food habits prior to surgery } \\
\text { Aim to introduce as much food variety as possible without compromising } \\
\text { nutrition and the healing process }\end{array}$ \\
\hline Frequency and size of meals & $\begin{array}{l}\text { Drastic changes in rate and level of food } \\
\text { intake may upset the body's metabolic } \\
\text { system especially after surgery } \\
\text { The frequency and size of meals are } \\
\text { determined by patient's tolerance and } \\
\text { comfort level }\end{array}$ & $\begin{array}{l}\text { Educate the patient to return to a regular eating schedule as soon as possible } \\
\text { Aim to establish an eating schedule that achieves adequate nutritional intake } \\
\text { Consider pre-existing medical conditions e.g. diabetes }\end{array}$ \\
\hline Ease of preparation & $\begin{array}{l}\text { Food choices that are more readily } \\
\text { available and easier to prepare would } \\
\text { encourage good food selection choices } \\
\text { and healthy eating habits that may opti- } \\
\text { mize healing }\end{array}$ & $\begin{array}{l}\text { Suggest the patient to prepare certain foods prior to surgery for the day(s) im- } \\
\text { mediately post-operation for the ease of consumption } \\
\text { Encourage the patient to establish a support network of friends and family to } \\
\text { assist with food preparation } \\
\text { Propose preparation methods that require little steps and technique } \\
\text { Offer referrals for nutritional counseling }\end{array}$ \\
\hline Fluid intake & $\begin{array}{l}\text { Dehydration may impede recovery, and } \\
\text { this may be exacerbated by vomiting. } \\
\text { Patients on certain medications have a } \\
\text { greater risk of developing xerostomia. }\end{array}$ & $\begin{array}{l}\text { Educate the patient on the importance of being well hydrated } \\
\text { Consider pre-existing medical conditions and interactions with medications }\end{array}$ \\
\hline
\end{tabular}

requires further study before specific recommendations can be made. Moreover, there is practical guidance, detailed in Table 4, which can assist patients with dietary choices postsurgery. This practical guidance includes considering the ease of intake of foods, ensuring that the food to be consumed will not damage or aggravate the wound site, as well as the palatability of the foods. Other considerations include the effort involved with preparing the recommended meals. If meals are too complicated and time-consuming to prepare, patients are less likely to be compliant. It is also important to educate the patient that they should return to a regular eating schedule as soon as possible. Because dehydration may impede recovery, it is important to ensure ample fluid intake. While developing an ideal dietary strategy to optimize wound healing is not often included in patient management, this review highlights specific aspects that could be considered to benefit the long-term periodontal health of a patient.

\section{CONFLICT OF INTEREST}

The authors confirm that this article content has no conflicts of interest.

\section{ACKNOWLEDGEMENT}

Wendy E. Ward holds a Canada Research Chair in Bone and Muscle Development.

\section{REFERENCES}

[1] Raja S, Byakod G, Pudakalkatti P. Growth factors in periodontal regeneration. Int J Dent Hyg 2009; 7(2): 82-9.

[2] Barroso AB, Lima V, Guzzo GC, et al. Efficacy and safety of combined piroxicam, dexamethasone, orphenadrine, and cyanocobalamin treatment in mandibular molar surgery. Braz J Med Biol Res 2006; 39(9): 1241-7.

[3] Zimmermann $\mathrm{T}$, Leonhardt $\mathrm{H}$, Kersting $\mathrm{S}$, et al. Reduction of postoperative lymphedema after oral tumor surgery with sodium selenite. Biol Trace Elem Res 2005; 106(3): 193-203. 
[4] Neiva RF, Al-Shammari K, Nociti FH, Jr., Soehren S, Wang HL. Effects of vitamin-B complex supplementation on periodontal wound healing. J Periodontol 2005; 76(7): 1084-91.

[5] Bashutski JD, Eber RM, Kinney JS, et al. The impact of vitamin D status on periodontal surgery outcomes. J Dent Res 2011; 90(8): 1007-12.

[6] Vieth R, Chan PC, MacFarlane GD. Efficacy and safety of vitamin D3 intake exceeding the lowest observed adverse effect level. Am J Clin Nutr 2001; 73(2): 288-94.

[7] Miley DD, Garcia MN, Hildebolt CF, et al. Cross-sectional study of vitamin D and calcium supplementation effects on chronic periodontitis. J Periodontol 2009; 80(9): 1433-9.

[8] Krall EA, Wehler C, Garcia RI, Harris SS, Dawson-Hughes B. Calcium and vitamin D supplements reduce tooth loss in the elderly. Am J Med 2001; 111(6): 452-6.

[9] Dietrich T, Joshipura KJ, Dawson-Hughes B, Bischoff-Ferrari HA. Association between serum concentrations of 25-hydroxyvitamin D3 and periodontal disease in the US population. Am J Clin Nutr 2004; 80(1): 108-13.

[10] Karjalainen S, Sewon L, Soderling E, et al. Oral health of 3-yearold children and their parents after 29 months of child-focused antiatherosclerotic dietary intervention in a prospective randomized trial. Caries Res 1997; 31(3): 180-5.

[11] Iwasaki M, Manz MC, Moynihan P, et al. Relationship between Saturated Fatty Acids and Periodontal Disease. J Dent Res 2011; 90(7): 861-7.

[12] Rosenstein ED, Kushner LJ, Kramer N, Kazandjian G. Pilot study of dietary fatty acid supplementation in the treatment of adult periodontitis. Prostaglandins Leukot Essent Fatty Acids 2003; 68(3): 213-8.

[13] Chapkin RS, Kim W, Lupton JR, McMurray DN. Dietary docosahexaenoic and eicosapentaenoic acid: emerging mediators of inflammation. Prostaglandins Leukot Essent Fatty Acids 2009; 81(2-3): 187-91.

[14] Naqvi AZ, Buettner C, Phillips RS, Davis RB, Mukamal KJ. n-3 fatty acids and periodontitis in US adults. J Am Diet Assoc 2010; 110(11): 1669-75.

[15] Iwasaki M, Yoshihara A, Moynihan $\mathrm{P}$, et al. Longitudinal relationship between dietary $\omega-3$ fatty acids and periodontal disease. Nutrition 2010; 26(11-12): 1105-9.

[16] Iwasaki M, Taylor GW, Moynihan P, et al. Dietary ratio of n-6 to n-3 polyunsaturated fatty acids and periodontal disease in community-based older Japanese: a 3-year follow-up study. Prostaglandins Leukot Essent Fatty Acids 2011; 85(2): 107-12.

[17] Sidi AD, Ashley FP. Influence of frequent sugar intakes on experimental gingivitis. J Periodontol 1984; 55(7): 419-23.

[18] Merchant AT, Pitiphat W, Franz M, Joshipura KJ. Whole-grain and fiber intakes and periodontitis risk in men. Am J Clin Nutr 2006; 83(6): 1395-400.

[19] Krall EA, Garcia RI, Dawson-Hughes B. Increased risk of tooth loss is related to bone loss at the whole body, hip, and spine. Calcif Tissue Int 1996; 59(6): 433-7.
[20] Uhrbom E, Jacobson L. Calcium and periodontitis: clinical effect of calcium medication. J Clin Periodontol 1984; 11(4):230-41.

[21] Nishida M, Grossi SG, Dunford RG, et al. Calcium and the risk for periodontal disease. J Periodontol 2000; 71(7): 1057-66.

[22] Dixon D, Hildebolt CF, Miley DD, et al. Calcium and vitamin D use among adults in periodontal disease maintenance programmes. Br Dent J 2009; 206(12): 627-31.

[23] Al-Zahrani MS. Increased intake of dairy products is related to lower periodontitis prevalence. J Periodontol 2006; 77(2): 289-94.

[24] Dietrich T, Nunn M, Dawson-Hughes B, Bischoff-Ferrari HA. Association between serum concentrations of 25-hydroxyvitamin D and gingival inflammation. Am J Clin Nutr 2005; 82(3): 575-80.

[25] Meisel P, Schwahn C, Luedemann J, et al. Magnesium deficiency is associated with periodontal disease. J Dent Res 2005; 84(10): 937-41.

[26] Rogo E, Hodges K, Herzog A. Dentinal sensitivity: a natural mineral dietary supplement study. Int J Dent Hyg 2006; 4(3): 1228.

[27] Iwasaki M, Manz MC, Taylor GW, Yoshihara A, Miyazaki H. Relations of serum ascorbic acid and alpha-tocopherol to periodontal disease. J Dent Res 2012; 91(2): 167-72.

[28] Iwasaki M, Moynihan P, Manz MC, et al. Dietary antioxidants and periodontal disease in community-based older Japanese: a 2-year follow-up study. Public Health Nutr 2013; 16(2): 330-8.

[29] Liede KE, Haukka JK, Saxen LM, Heinonen OP. Increased tendency towards gingival bleeding caused by joint effect of alphatocopherol supplementation and acetylsalicylic acid. Ann Med 1998; 30(6): 542-6.

[30] Nishida M, Grossi SG, Dunford RG, et al. Dietary vitamin C and the risk for periodontal disease. J Periodontol 2000; 71(8): 121523.

[31] Amarasena N, Ogawa H, Yoshihara A, Hanada N, Miyazaki H. Serum vitamin C-periodontal relationship in community-dwelling elderly Japanese. J Clin Periodontol 2005; 32(1): 93-7.

[32] Woolfe SN, Kenney EB, Hume WR, Carranza FA, Jr. Relationship of ascorbic acid levels of blood and gingival tissue with response to periodontal therapy. J Clin Periodontol 1984; 11(3): 159-65.

[33] Lingstrom P, Fure S, Dinitzen B, et al. The release of vitamin C from chewing gum and its effects on supragingival calculus formation. Eur J Oral Sci 2005; 113(1): 20-7.

[34] Staudte H, Sigusch BW, Glockmann E. Grapefruit consumption improves vitamin C status in periodontitis patients. Br Dent J 2005; 199(4): 213-7.

[35] Chandra RV, Prabhuji ML, Roopa DA, Ravirajan S, Kishore HC. Efficacy of lycopene in the treatment of gingivitis: a randomised, placebo-controlled clinical trial. Oral Health Prev Dent 2007; 5(4): 327-36.

[36] Kushiyama M, Shimazaki Y, Murakami M, Yamashita Y. Relationship between intake of green tea and periodontal disease. $\mathrm{J}$ Periodontol 2009; 80(3): 372-7.

[37] Krahwinkel T, Willershausen B. The effect of sugar-free green tea chew candies on the degree of inflammation of the gingiva. Eur J Med Res 2000; 5(11): 463-7. 